potential to affect patient safety, with a minority of medical schools offering limited teaching and patient contact.

\title{
P 009 TEACHING PALLIATIVE CARE (PC) TO MEDICAL STUDENTS; ARE WE DEVELOPING SAFE PRACTITIONERS?
}

\author{
Steven Walker, ${ }^{1,2}$ Faye Gishen, ${ }^{1,3}$ Philip Lodge, ${ }^{1}$ Bee Wee ${ }^{4}$. ${ }^{1}$ Marie Curie \\ Hospice, London; ${ }^{2}$ University of Dundee; ${ }^{3}$ University College London; \\ ${ }^{4}$ Sir Michael Sobell House \& University of Oxford, UK \\ 10.1136/bmjspcare-2014-000838.12
}

Introduction Appropriate training is essential to ensure PC is safely practised ${ }^{1}$. A proportion of newly qualified doctors report that undergraduate teaching has not adequately prepared them to manage PC patients. ${ }^{2}$ Little is known about how PC training is delivered across UK medical schools ${ }^{3}$. Addressing this requires key data; one group likely to have an overview are the responsible PC educational leads.

Aim(s) and method(s) To undertake a comprehensive review of PC teaching across UK medical schools by means of an anonymised, multifactorial questionnaire of educational leads.

A 40-point questionnaire was developed using SurveyMonkey ${ }^{\circledR}$ and sent to PC educational leads at all 30 UK medical schools. This study received ethical approval.

Results The response was 100\%. PC teaching occurred in all institutions. In 20 (67\%), there was a formal PC component. Teaching around last days of life, death and bereavement was mandatory. Training in other areas (e.g. emergencies) was sometimes optional. Teaching was generally 'integrated' (15 respondents) and/or 'formed a module' within a larger course (17 respondents) and generally occurred in the final 2 years. A student selected component was offered by 27 (90\%).

Most respondents were positive about their courses which, in general, were well supported locally, personally satisfying and highly rated by students.

\section{Concerns included:}

- Limited student placements $(20,66 \%)$.

- Restricted opportunity to visit a hospice and observe MDT care (10, 33\%)

- Insufficient teachers $(22,73 \%)$

- Insufficient funding $(10,33 \%)$

- Variability in teaching $(15,50 \%)$ Whether courses prepared doctors to care for PC patients $(9,30 \%)$ delivered quality training $(5,17 \%)$ and fulfilled GMC requirements $(2,7 \%)$.

Conclusions PC training for medical students in the UK continues to evolve with greater integration into the curriculum and the wider use of learning assessment. There is, however, variability, which has the 